\title{
Simple Interrupted Microvascular Anastomosis: Review of Four Sutures Placement Sequences
}

\author{
Brianne Sullivan, MD ${ }^{1}$ Michael Zoppo, BA ${ }^{2} \quad$ Alice Yao, MD ${ }^{1}$ Peter W. Henderson, MD, MBA, FACS ${ }^{1}$ \\ ${ }^{1}$ Division of Plastic and Reconstructive Surgery, Department of \\ Surgery, Icahn School of Medicine at Mount Sinai, New York, \\ New York \\ 2 BlueTeam Design, Raleigh, North Carolina \\ Address for correspondence Peter W. Henderson, MD, MBA, FACS, \\ Division of Plastic and Reconstructive Surgery, Department of \\ Surgery, Icahn School of Medicine at Mount Sinai, 10 Union Square \\ East, Suite 2L, New York, NY 10003 \\ (e-mail: peter.henderson@mountsinai.org).
}

| Reconstr Microsurg Open 2020;5:e101-e106.

\begin{abstract}
Keywords

- microvascular anastomosis

- simple interrupted

- microvascular anastomosis technique

- microsurgery

Background Microsurgical anastomosis is a delicate, highly nuanced procedure that can be done in several different ways; the most basic approach to performing microsurgical anastomoses is by using simple interrupted sutures, but even within that approach, there are different sequences by which the sutures can be placed. To date, there is no review of these sequences and the advantages and disadvantages of each.

Methods A PubMed search was performed in October 2019 that sought all published descriptions of simple interrupted microsurgery techniques. Keywords included were "microvascular anastomosis," "microvascular anastomosis technique," "simple interrupted," and "microsurgery."

Results Four simple interrupted microsurgery techniques were identified: triangulation, 12 o'clock to 6 o'clock, posterior-wall-first, and 3 o'clock to 9 o'clock-side-side. Additionally, there is no uniform nomenclature that describes the techniques for simple interrupted microvascular anastomoses.

Conclusion This study identified four distinct sequences for the placement of simple interrupted sutures for microsurgical anastomosis and the advantages and disadvantages of each, and for the first time described them utilizing standardized nomenclature.
\end{abstract}

Successful performance of a microsurgical anastomosis is imperative in free tissue transfer and limb/digit replantation. It is a delicate and highly nuanced procedure that can be done in several ways. There is a wide range of microvascular anastomosis techniques described in the literature with the optimal technique being a topic of debate. Some of the options for performing a microsurgical anastomosis include simple interrupted, continuous, locking continuous, interrupted horizontal mattress, sleeve anastomosis, fibrin glue, couplers, and microclips. ${ }^{1}$ The ideal microvascular anastomosis technique would be easy to perform, minimize ischemia time, avoid vessel wall trauma, and lead to both shortand long-term patencies. It is widely understood that the simple interrupted suture technique is the most standard and easily conceptualized approach to microvascular anastomosis. ${ }^{1-4}$ It is less widely understood, however, that there are many different sequences by which simple interrupted sutures can be placed.

There are certain clinical variables that influence whether one sequence is more or less preferable (these include length of vessel, surgical skill level, room for microvascular clamps, and availability of an assistant). ${ }^{4-7}$ Such nuances of each sequence have been largely ignored in the literature, as descriptions of the suture sequence is often ignored all together, and there is varied nomenclature. The purpose of this study is to identify every distinct suture placement received

June 23, 2020 accepted after revision August 25, 2020
DOI https://doi.org/

10.1055/s-0040-1719152. ISSN 2377-0813.
Copyright $\odot 2020$ by Thieme Medical Publishers, Inc., 333 Seventh Avenue, New York, NY 10001, USA. Tel: +1(212) 760-0888.
License terms

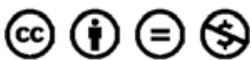




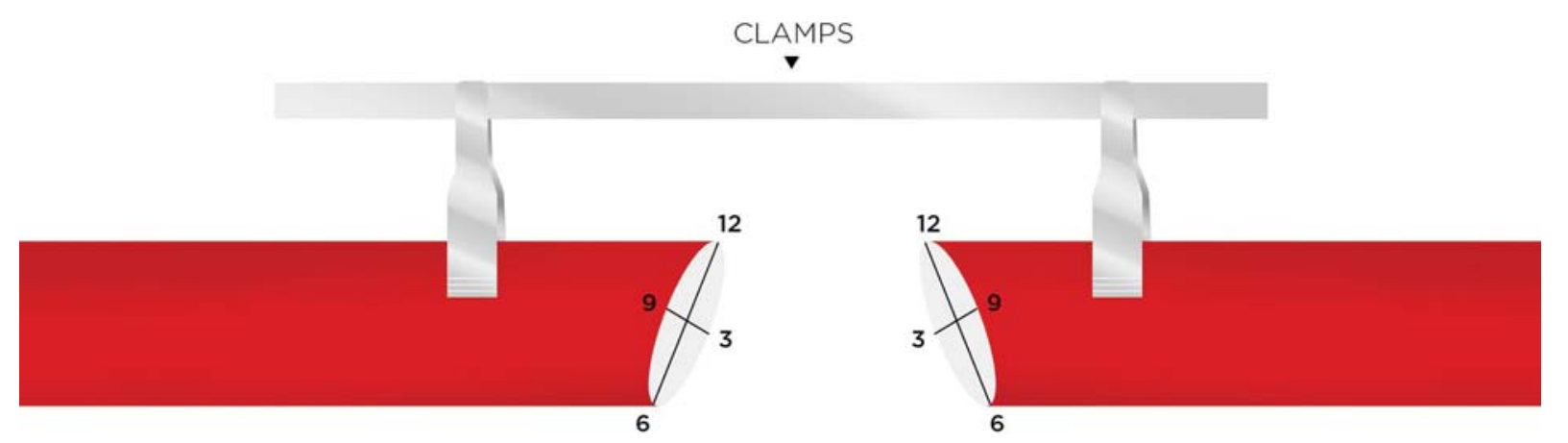

Fig. 1 Orientation of transected blood vessel in relation to the patient and operating clamps.

sequence for microvascular anastomosis published in the literature, describe them using standardized nomenclature, and discuss the clinical scenarios in which each technique is the most appropriate.

\section{Methods}

A PubMed search was performed in October 2019 using the keywords "microvascular anastomosis," "microvascular anastomosis technique," "simple interrupted," and "microsurgery" to identify the types of simple interrupted microvascular anastomoses described in the literature.

Additionally, we created a standardized nomenclature by demonstrating the orientation of the blood vessel in a patient with surgical clamps and assigning clock face values to a transected blood vessel (-Fig. 1). We define 9 o'clock as the most anterior part of the blood vessel and 3 o'clock as the most posterior ("deepest") part of the blood vessel. Based on this, we define 12 o'clock as the midpoint of the segment farther from the surgeon between 9 o'clock and 3 o'clock, and 6 o'clock as the midpoint of the segment closer to the surgeon between 3 o'clock and 9 o'clock. The anterior wall is the 180 degrees of the vessel that runs from 6 o'clock to 9 o'clock to 12 o'clock, and the posterior wall is the 180 degrees of the vessel that runs from 6 o'clock to 3 o'clock to 12 o'clock. The sidewalls of the vessel run between 9 o'clock and 3

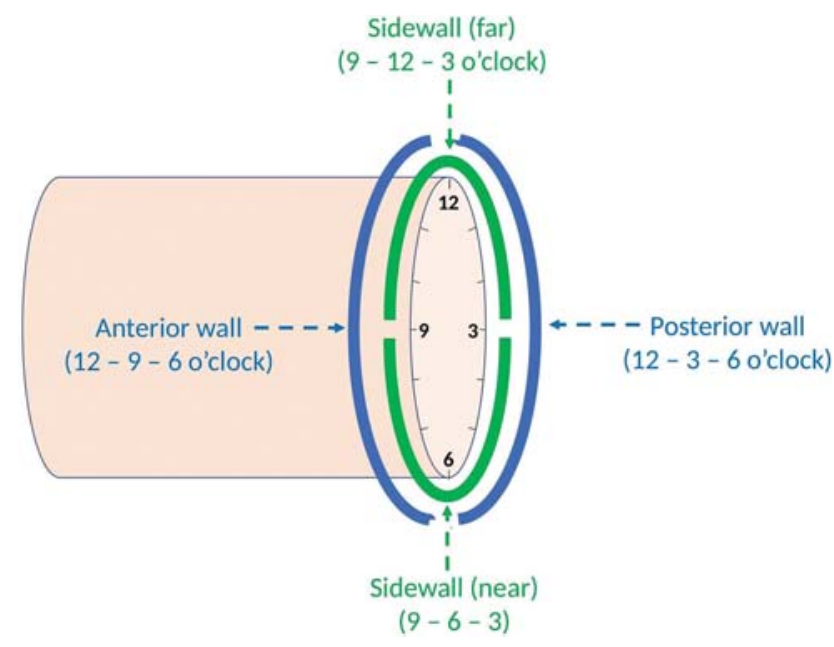

Fig. 2 Standardized nomenclature of a transected blood vessel. o'clock, with the near sidewall as the one closer to the surgeon, and the far sidewall as the one farther from the surgeon (-Fig. 2).

By this nomenclature, anterior wall, posterior wall, and the two sidewalls are each 180 degrees of the vessel circumference. Therefore, the logic must follow that anterior/ posterior walls and sidewalls cannot coexist in the same scheme. When the first sutures are placed, they define whether the vessel circumference will be conceptualized as either anterior/posterior walls or sidewalls, and this will remain constant for the entirety of the suture sequence.

\section{Results}

Four distinct simple interrupted microsurgical anastomosis techniques were identified: triangulation, 12 o'clock to 6 o'clock, posterior-wall-first, and 3 o'clock to 9 o'clock.

\section{Triangulation}

First described by Alexis Carrel in 1902, the triangulation technique was one of the first methods to be used in microvascular repair of small vessels, and was the basis for his being awarded the Nobel Prize in $1912 .{ }^{4}$ The triangulation technique is still referred to in some articles as the "classical" microanastomosis, ${ }^{8}$ but the term triangulation is inconsistently used. ${ }^{9}$

In the triangulation technique, three stay sutures are placed 120 degrees apart, at the 11 o'clock, 3 o'clock, and 7 o'clock positions (- Fig. 2). Tension is applied to two of the sutures, lifting the wall being sutured away from the underlying wall. ${ }^{4}$ Two additional sutures are placed between each pair of stay sutures. ${ }^{10}$ Approximating double-opposing microvascular clamps are usually used.

The key advantage of the triangulation technique is that the asymmetrical placement of the stay sutures minimizes the risk of inadvertently incorporating the intima of the back wall, since tension on the two stay sutures raises the segment of wall being sutured up from the longer underlying wall, thereby opening the lumen. Triangulation technique is technically more challenging when compared with anterior wall first techniques. Theoretically, vessel wall mismatch may occur when attempting to gauge 120 degrees. However, Kim et al actually showed more regular suture placement when comparing the triangulation technique with the 12 o'clock to 6 


\section{TRIANGULATION}

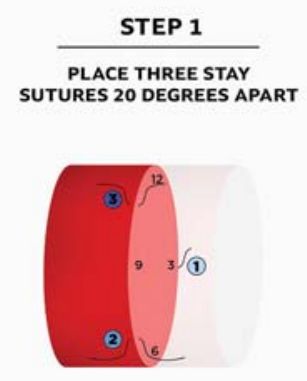

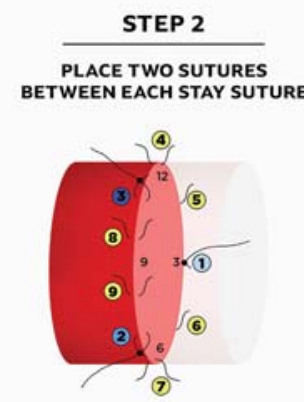

Fig. 3 Triangulation microvascular anastomosis technique.

o'clock technique in a small randomized controlled trial, likely secondary to more sutures being placed in the triangulation technique reducing interstitch distance and thus variability in placement. ${ }^{4}$ That being said, typically, the more sutures that need to be placed are considered a risk for stenosis and/or thrombosis. ${ }^{1}$ Clinically, the triangulation technique may be useful for trainees as one of the biggest advantages and reduces the chance of including the back wall.

\section{2 o'Clock to 6 o'Clock}

Jacobson and Suarez ${ }^{11}$ are largely credited for the development of microvascular surgery in 1960 when they successfully began to anastomose vessels less than $1 \mathrm{~mm}$ in diameter. ${ }^{12}$ The operating microscope, in addition to new suture and instruments, allowed for precision and new techniques in manual suture. ${ }^{13}$ The first description of the 12 o'clock to 6 o'clock microvascular surgery technique is difficult to find in the literature but is presumed to have originated during this time period. This technique is considered the most basic and is most often referred to as the conventional method to perform a simple interrupted microvascular anastomosis. ${ }^{2,4}$

In this method, the first suture is placed at 12 o'clock (also sometimes referred to as 0 degree), and the second stitch is placed at 6 o'clock (or 180 degrees). The third and fourth sutures are placed at 2 o'clock and 4 o'clock completing the anterior wall. The vessel is then turned over 180 degrees with a clamp, and the fifth and sixth sutures are placed at 8 o'clock and 10 o'clock (-Fig. 3). ${ }^{6}$ Cobbett revised the standard 12 o'clock to 6 o'clock technique with the "eccentric biangulation" method where the first two sutures are placed slightly more anterior to the 12 o'clock and 6 o'clock positions. Double-opposing microvascular clamps are necessary to rotate the vessel.

The major disadvantage of this technique is the need to rotate the vessel 180 degrees to suture the posterior wall. This requires enough length on the vessel and room for the clamps, and introduces the potential vessel damage during the rotation. This technique may also be beneficial for trainees learning microsurgery as it is technically most simple as long as space for the clamps and rotation can be obtained.

\section{Posterior Wall First}

The technique, advantages, and outcomes of the posteriorwall-first technique were first described by Harris et al in 1981. ${ }^{5}$ This method is also sometimes referred to as the "back-up" technique.

The first suture is placed in the middle of the posterior wall with the second and third sutures placed on either side of the first ( - Fig. 4). The fourth and fifth sutures are placed adjacent to the second and third advancing anteriorly on either side, leaving a long tail to facilitate placement of the

\section{“12 O’CLOCK, 6 O’CLOCK, FRONT WALL, BACK WALL”}

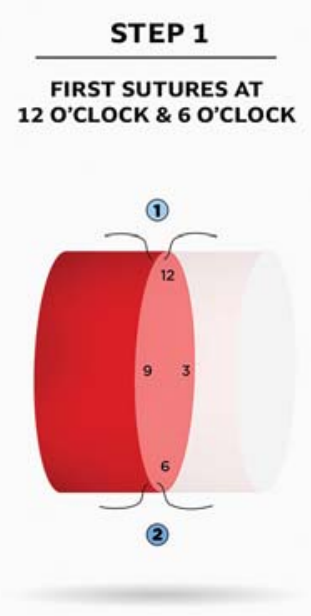

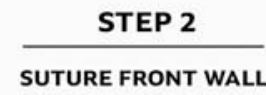

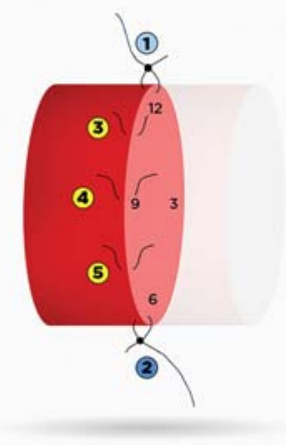

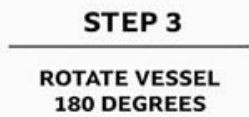

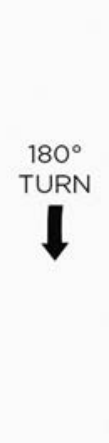

180 DEGREES

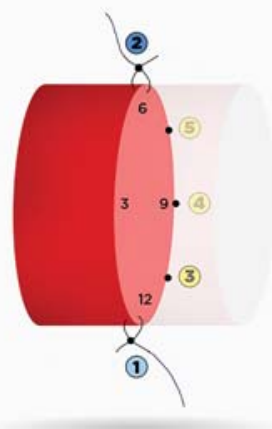

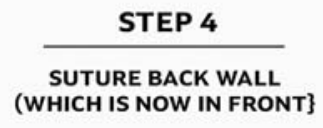

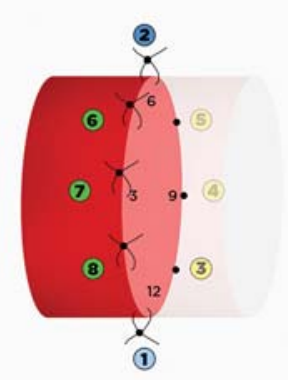

Fig. 4 The 12 o'clock to 6 o'clock microvascular anastomosis technique. 
sixth and seventh sutures. In a similar fashion, both the sixth and seventh sutures are left long to aid placement of the last suture, which is placed equidistant from the sixth and the seventh. Double-opposing microvascular clamps are not required for this anastomosis as there is no rotation of the vessels.

Harris et al listed many advantages and declared there are no disadvantages to the posterior-wall-first technique. Advantages include constant visualization of the back wall decreasing the chance of accidentally catching the back wall, particularly useful for microsurgeons in training. There is no need to rotate clamps, allowing placement of sutures in spaces where maneuvering clamps is difficult or impossible. The authors also consider the technique mentally less stressful as the surgeon does not lose concentration to reposition, and they know each subsequent stitch is easier than the one prior. Ultimately, the authors conclude the posterior-wallfirst technique is less complicated, more rapid, and easier to perform compared with anterior-wall techniques. ${ }^{5}$

Although Harris et al do not report any disadvantages, other authors have noted that operating on the back wall may be technically more challenging and time consuming. ${ }^{8}$ Clinically, this technique is advantageous in scenarios where maneuvering clamps is difficult or impossible, for instance, a venous anastomosis during digital replantation, a short recipient vessel leash, or friable/radiated vessels. Additionally, it may be useful when there is a size mismatch between the vessels being anastomosed.

\section{3 o'Clock to 9 o'Clock-Side-Side}

Yu et al published, in 1986, a description of a simplified technique for end-to-end microanastomosis that involved placing the first suture through the posterior wall, second suture through the anterior wall with 90 degrees of rotation to facilitate the placement of the remaining sutures. $^{8}$

In the technique, clamps are placed opposite the surgeon and the first suture is placed in the center of the posterior wall (-Fig. 5). The second suture is placed in the center of the anterior wall, 180 degrees to the first suture. Both sutures are left with long tails. A new anterior wall is created by bringing the tail of the posterior wall superiorly and the tail of the anterior wall inferiorly, rotating the vessel 90 degrees. The third, fourth, and fifth sutures are placed along the new anterior wall. The vessel is then rotated back to its original position and once again traction is placed to rotate another 90 degrees bringing the unsutured half of the vessel anteriorly and sutures 6,7 , and 8 are placed. ${ }^{8}$

According to the authors, the benefits of this technique is that it allows the surgeon to place only one suture in the posterior wall, while all other sutures are placed in the wall facing the surgeon. The angle of rotation is less than the conventional 180 degrees, decreasing possible damage to the endothelium and decreasing the need to dissect the vessels proximal and distal to the clamps. Additionally, the authors propose this method is technically easier to perform than posterior-wall-first technique. ${ }^{8}$

Nevertheless, one suture is still placed in the posterior wall, which is more challenging than placing all sutures on the wall facing the surgeon. This technique is particularly useful in settings with short vessel segments, when one may not want to ligate branching vessels to increase vessel length or rotating the vessel is difficult due to space constraints (-Fig. $\mathbf{6}$ ).

\section{Discussion}

The birth of microsurgery dates back to 1906 when Alexis Carrel first described a small vessel anastomosis. ${ }^{12,14}$ The

\section{POSTERIOR WALL FIRST}
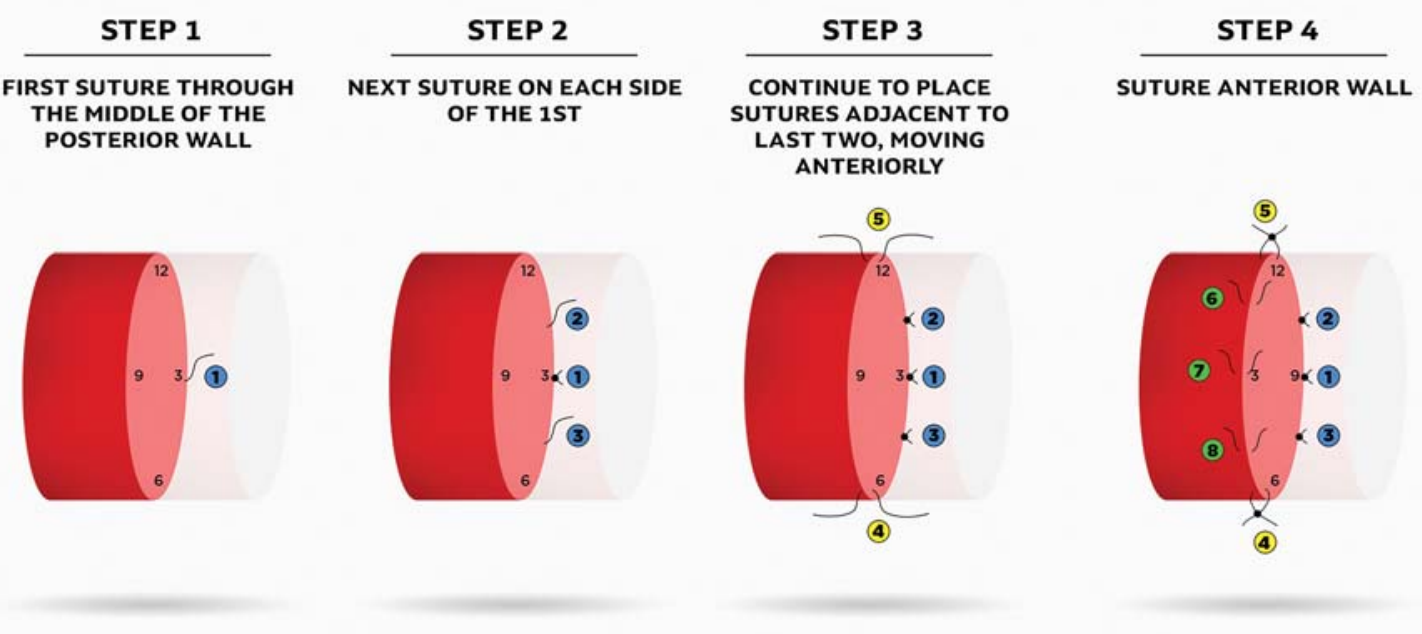

Fig. 5 Posterior-wall-first microvascular anastomosis technique. 


\section{O'CLOCK - 9 O'CLOCK}

STEP 1

SUTURE CENTER OF

POSTERIOR AND

ANTERIOR WALL

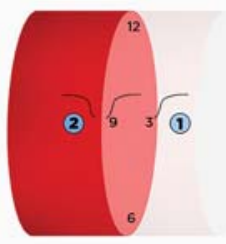

STEP 2

ROTATE 90 DEGREES.

SUTURE SIDE WALL

(WHICH IS NOW IN FRONT)

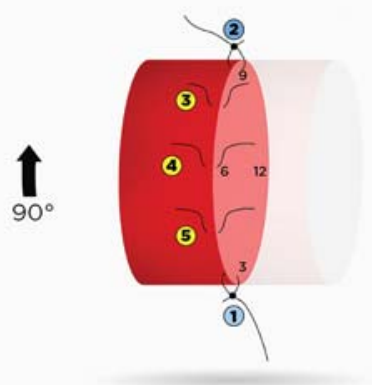

STEP 3

RETURN TO NEUTRAL,

ROTATE 90 DEGREES

IN THE OTHER DIRECTION,

(1)

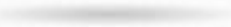

Fig. 6 The 3 o'clock to 9 o'clock-side-side microvascular anastomosis technique.

next major advance was not until 1960 when Jacobson and Suarez anastomosed $1 \mathrm{~mm}$ vessels and the microscope was utilized in the operating room. ${ }^{15}$

Descriptions of how simple interrupted microsuture anastomoses are performed are often vague in research articles. Firsching et $\mathrm{al}^{16}$ compared continuous to simple microsuture technique and described continuous methods in detail but did not describe the simple anastomosis method at all. Alghoul et al and Turan et al both reported the simple interrupted microvascular suture is the conventional technique and "gold standard," but Turan et al described the conventional technique as the triangulation method, while Alghoul et al considered the conventional technique to encompass both the 12 o'clock to 6 o'clock and the triangulation methods. ${ }^{1,17}$ There were also clear discrepancies in the literature when authors apply clock face values to the vessel walls. Most frequently, 9 o'clock is used to denote the most anterior aspect of the transected blood vessel, but one article

Table 1 Advantages and disadvantages of each simple interrupted microvascular suture technique

\begin{tabular}{|l|l|l|l|l|}
\hline & Triangulation & $\mathbf{1 2}$ o'clock-6 o'clock & Posterior-wall-first & $\mathbf{3}$ o'clock-9 o'clock \\
\hline $\begin{array}{l}\text { Required } \\
\text { instruments }\end{array}$ & $\begin{array}{l}\text { Double-opposing } \\
\text { microvascular clamp }\end{array}$ & $\begin{array}{l}\text { Double-opposing } \\
\text { microvascular clamp }\end{array}$ & None & $\begin{array}{l}\text { Double-opposing } \\
\text { microvascular } \\
\text { clamp or assistant }\end{array}$ \\
\hline $\begin{array}{l}\text { Degrees of } \\
\text { rotation }\end{array}$ & 120 & 180 & 0 & 90 \\
\hline Advantages & $\begin{array}{l}\text { Reduces chance of } \\
\text { posterior wall injury }\end{array}$ & Technically simpler & $\begin{array}{l}\text { Optimal when there are } \\
\text { space limitations (minimal } \\
\text { vessel length needed) }\end{array}$ & $\begin{array}{l}\text { No extensive dissection of } \\
\text { vessel required proximal or } \\
\text { distal to clamps }\end{array}$ \\
\cline { 2 - 5 } & & $\begin{array}{l}\text { Most commonly } \\
\text { performed }\end{array}$ & $\begin{array}{l}\text { Reduces chance of posterior } \\
\text { wall injury } \\
\text { (direct visualization) }\end{array}$ & $\begin{array}{l}\text { Less rotation can cause } \\
\text { less intimal damage }\end{array}$ \\
\cline { 2 - 5 } & $\begin{array}{l}\text { Technically more } \\
\text { challenging }\end{array}$ & $\begin{array}{l}\text { Requires greater } \\
\text { length of vessel } \\
\text { exposure (to allow for } \\
180 \text { deg rotation) }\end{array}$ & $\begin{array}{l}\text { Technically more challenging } \\
\text { (posterior wall visualization) }\end{array}$ & Requires assistant \\
\cline { 2 - 5 } & $\begin{array}{l}\text { Challenge to match } \\
\text { vessel edges }\end{array}$ & & More time consuming & \\
\hline
\end{tabular}


assigned 12 o'clock as the most anterior aspect of the vessel. ${ }^{18}$ Yet another author used degrees to describe location around the vessel, 0 and 180 degrees representing the most anterior and posterior points, respectively. ${ }^{19}$

Upon reviewing each simple interrupted microvascular suture technique, it is apparent that each has advantages and disadvantages (-Table 1). Surgeons may find themselves in clinical scenarios where some techniques may be favorable over others. In summary, the most commonly performed is the 12 o'clock to 6 o'clock, especially for beginners who are just beginning to visualize the threedimensional structure of the vessel. Posterior-wall-first and triangulation techniques may lead to lower risk of catching the back wall in difficult cases or time-sensitive situations. If presented with a short vessel leash, limited operative space, or injured/friable vessels, one may consider the posterior-wall-first or the 3 o'clock to 9 o'clock-side-side techniques to avoid the use of Acland clamps or excessive torsion on the vessels. It would be beneficial for the experienced microsurgeon to become familiar with all available techniques to optimize their operative field based on each unique case.

Research is lacking comparing the simple interrupted techniques. There is one randomized controlled study that compares the advantages and disadvantages of the triangulation technique to the "biangulation" technique, or as we refer to it the 12 o'clock to 6 o'clock, to find quantitative evidence for which technique should be advocated to incorporate in microsurgery training. ${ }^{4}$ In the study, medical students and surgical trainees underwent a 5-day microsurgery course. Their ability to perform a biangulation and triangulation anastomoses was evaluated on days 3 and 5 with the order of technique performed randomized. The outcomes of the two techniques in terms of vessel structural patency and quality of anastomosis were not significantly different, thus the authors concluded that both techniques are equally appropriate for surgical training. ${ }^{4}$

Additional studies that further compare the four techniques in the clinical setting are currently underway at our institution and will be reported in future publications.

\section{Conclusion}

This study identified four distinct sequences for the placement of simple interrupted sutures for microsurgical anastomosis, and for the first time described them in a standardized nomenclature.

\section{Conflict of Interest}

None declared.

\section{Acknowledgment}

We would like to thank BueTeam Design for collaborating and using their expertise with graphic design to help create the figures.

\section{References}

1 Alghoul MS, Gordon CR, Yetman R, et al. From simple interrupted to complex spiral: a systematic review of various suture techniques for microvascular anastomoses. Microsurgery 2011;31(01): $72-80$

2 Hou SM, Seaber AV, Urbaniak JR. An alternative technique of microvascular anastomosis. Microsurgery 1987;8(01):22-24

3 Agko M, Ciudad P, Chen HC. "Airborne" suture tying technique: simple steps to make it easy. Head Neck 2017;39(12):2558-2561

4 Kim E, Singh M, Akelina Y, Shurey S, Myers SR, Ghanem AM. Effect of microvascular anastomosis technique on end product outcome in simulated training: a prospective blinded randomized controlled trial. J Reconstr Microsurg 2016;32(07):556-561

5 Harris GD, Finseth F, Buncke HJ. Posterior-wall-first microvascular anastomotic technique. Br J Plast Surg 1981;34(01):47-49

6 Hou SM, Seaber AV, Urbaniak JR. An alternative technique of microvascular anastomosis. Microsurgery 1987;8(01):22-24

7 Chen ZW, Yang DY, Chang DA. Microsurgery Shanghai Scientific and Technical Publishers. Berlin; New York: Springer-Verlag; 1982

8 Yu HL, Sagi A, Ferder M, Strauch B. A simplified technique for endto-end microanastomosis. J Reconstr Microsurg 1986;2(03): 191-194

9 Lee BY, Thoden WR, Brancato RF, Kavner D, Shaw W, Madden JL. Comparison of continuous and interrupted suture techniques in microvascular anastomosis. Surg Gynecol Obstet 1982;155(03): 353-357

10 Tetik C, Ünal MB, Kocaoğlu B, Erol B. Use of continuous horizontal mattress suture techniques in microsurgery: an experimental study in rats. J Hand Surg Am 2005;30(03):587-595

11 Jacobson JH, Suarez EL. Microsurgery in Anastomosis of Small Vessels. Surg Forum 1960;11:243

12 Gordon L. Advances in microsurgery. Surg Technol Int 1991; I:425-427

13 Cobbett JR. Microvascular surgery. Surg Clin North Am 1967;47 (02):521-542

14 Rickard RF, Hudson DA. A history of vascular and microvascular surgery. Ann Plast Surg 2014;73(04):465-472

15 Jacobson JH II. The early days of microsurgery in Vermont. Mt Sinai J Med 1997;64(03):160-163

16 Firsching R, Terhaag PD, Müller W, Frowein RA. Continuous and interrupted suture technique in microsurgical end?to? end anastomosis Microsurgery 1984;5:80-84

17 Turan T, Ozçelik D, Kuran I, et al. Eversion with four sutures: an easy, fast, and reliable technique for microvascular anastomosis. Plast Reconstr Surg 2001;107(02):463-470

18 Chen YX, Chen LE, Seaber AV, Urbaniak JR. Comparison of continuous and interrupted suture techniques in microvascular anastomosis. J Hand Surg Am 2001;26(03):530-539

19 Barros RSM, Leal RA, Teixeira RKC, et al. Continuous versus interrupted suture technique in microvascular anastomosis in rats. Acta Cir Bras 2017;32(09):691-696 